\title{
Modified control chart for monitoring the variance*
}

\author{
Tiago Ruckert Landim ${ }^{1}$, Felipe Schoemer Jardim², Pedro Carlos Oprime ${ }^{1}$ \\ ${ }^{1}$ Federal University of São Carlos - UFSCAR, São Carlos, SP, Brazil. \\ 2Fluminense Federal University - UFF, Niterói, RJ, Brazil
}

How to cite: Landim, T.R., Jardim, F.S. and Oprime, P.C. (2021), "Modified control chart for monitoring the variance", Brazilian Journal of Operations \& Production Management, Vol. 18, No. 03, e20211129. https://doi.org/10.14488/BJOPM.2021.015

\begin{abstract}
Goal: The main objective of this research paper is to propose a chart, named $S^{2}$ Modified Control Chart, where the process variance $\left(\sigma^{2}\right)$ is allowed to be larger than the in-control variance value $\left(\sigma_{0}^{2}\right)$ until a maximum value ( $\sigma_{M A X}^{2}$ ), as long as the process remains capable, in the sense that it produces a specified (tolerated) small fraction of non-conforming items.

Design / Methodology / Approach: The research methodology was quantitative approach with statistical analysis of simulated data, to assess the practical impact of variance increase in process control in terms of quality requirements.

Results: The analysis of the simulated data showed that by using the proposed $S^{2}$ Modified Control Chart the number of unnecessary interventions in the process could be decreased, contributing to improve its efficiency.

Limitations of the investigation: The analysis assumed mean and variance known, which is unlikely to occur in real applications. Research considering unknown parameters is also in progress.

Practical implications: The $\mathrm{S}^{2}$ Modified Control Chart detects only genuinely increases in the process variance, which significantly increase the rate of non-conforming items being produced, preventing unnecessary process stop and assessment for assignable causes if only a small increase in the process variance occurs, contributing for higher process efficiency and reduce costs.

Originality / Value: This paper introduces a new control chart to monitor the variance of quality characteristics being produced by capable processes, preventing process overcontrol.

Keywords: Type I and Type II Errors; False Alarm Rate; Acceptance and Modified Control Chart; S² Control Chart.
\end{abstract}

\section{INTRODUCTION}

Control charts are powerful tools used by many industries to monitor the quality of processes and detect special cause of variations on them. The $S^{2}$ Control Chart is one of the most used tools to monitor if the variance of some quality characteristic $(X)$, which is assumed to be normally distributed, may change from an in-control (IC) to an out-of-control (OCC) situation. The main objective of this chart is to detect increases of any magnitude in the

${ }^{*}$ This article is from the XXVI International Conference on Industrial Engineering and Operations Management (IJCIEOM) and was invited to be published in the September/2021 Special Issue of the Brazilian Journal of Operations \& Production Management, due to its relevance and contribution to the field.

Financial support: None.

Conflict of interest: The authors have no conflict of interest to declare.

Corresponding author: tiagolandim@gmail.com

Received: 21 Dec 2020

Approved: 4 Jan 2021

Editor: Rafael Garcia Barbastefano 
process variance, as soon as possible. In this context, if the actual process variance is larger than an in-control single level point, the process is considered to be in an out-of-control state.

The basic procedure of the $S^{2}$ Control Chart is the following: samples of size $n$ (of some quality characteristic, $X$, of the product being produced) are collected at regular intervals so the sample variance $\left(s^{2}\right)$ can be computed. This sample variance is compared with a control limit. If $S^{2}$ is above the control limit, chances should be high that the process is out of control, or in other words, chances should be high that the actual process variance is larger than the nominal in-control value.

However, in some situations, even if a process is declared out of control, it might still be capable from a practical point of view in the sense that it still produces an acceptably low proportion of non-conforming items and hence the process does not need to be stopped in order to look for assignable causes. This can save valuable time and resources. In other words, if the process variance is allowed to be a bit larger than the in-control variance value and yet the rate of non-conforming items being produced is small enough, this may be a tolerable situation from a practical point of view.

In summary, it is of interest to monitor the process mean and variance with control charts with a broader definition of "in-control" together with the capability of the process. Unfortunately, the original Shewhart $\bar{X}$ and $S^{2}$ control charts are not designed for this type of monitoring. Instead, in this situation, the Modified and the Acceptance charts, (which are Shewhart-type charts) introduced respectively by Hill (1956) and Freund (1957), are more appropriate tools, since they allow the process mean to vary between two specified/tolerated limits (Montgomery, 2009) and yet ensure that only a small proportion of non-conforming items are produced so there is no need to declare the process out-of-control and start a search for assignable causes.

Modified and Acceptance charts are also powerful tools to avoid many false alarms and this is especially important nowadays when there are several systems with many control charts being used simultaneously, as emphasized recently by Woodall and Faltin (2019). Modified (and Acceptance) control charts generate less false alarms (compared with the Shewhart $\bar{X}$ chart) because, as explained above, they are designed to detected only genuinely important changes in the process mean (changes that generate a rate of non-conforming items larger than what is specified). So, even though these charts were created a long time ago (in the 50's), they may be still of great value in practice today. More applications of these types of charts can be found in Mohammadian and Amiri (2012), Oliveira et al. (2018) and Wu (1998).

Unfortunately, the Modified and Acceptance Control charts were designed only focusing on monitoring the process mean. As emphasized by several authors, see for example Montgomery (2009), monitoring the process variance is also important to avoid the production of an undesirable amount of non-conforming units. Given this background as motivation, this work extent the idea of the Modified and Acceptance Control Charts by focusing on monitoring the process dispersion.

We emphases that the present paper is a part of a work in progress being developed by us where we also study the power of the $s^{2}$ Modified Control Chart and the case where it is designed with estimated parameters and applied jointly with the $\bar{X}$ Modified Control Chart.

The rest of the paper will be presented in four parts. First, we provide an overview of the well-know $s^{2}$ Control Chart emphasizing the problem of not considering the process capability in its formulation. In sequence, we present the model of the Modified Control Chart for monitoring the process variance, presenting the equation for its control limit. The third part brings an illustrative example (using simulation) of the application of the new proposed Modified Control Chart for monitoring the variance (a comparison with the $s^{2}$ control chart is also provided) and finally the conclusions. 


\section{A REVIEW OF THE $s^{2}$ CONTROL CHART}

The $S^{2}$ Control Chart is one of the most well-known tools to monitor the variance of a quality characteristic $(X)$ of processes in many industries. As presented in the Introduction, the main objective of the $s^{2}$ Control Chart is to detect increases (of any magnitude) in the process variance $\left(\sigma^{2}\right)$, as soon as possible. In this context, if the actual process variance $\left(\sigma^{2}\right)$ is larger (by any magnitude) than an in-control single level point $\left(\sigma_{0}^{2}\right)$, the process is considered to be in an out-of-control state, otherwise the process is declared in control. Figure 1 illustrates this situation.

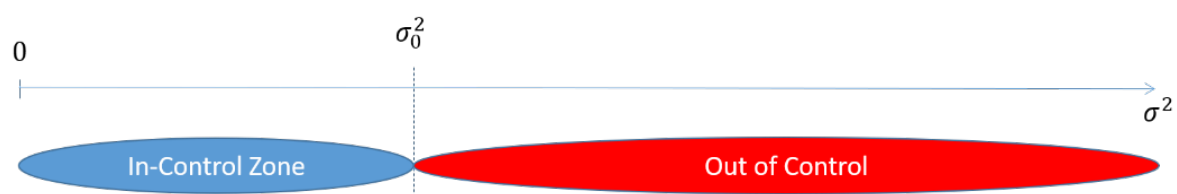

Figure 1. The In-Control Zone and Out-of-Control Zones of the $S^{2}$ Control Chart. Source: The author themselves.

This chart shall be used together with the mean control chart, in order properly detect changes for the mean. To monitor the process variance $\left(\sigma^{2}\right)$ with the $s^{2}$ Control Chart samples of size $n$ of the quality characteristic $(X)$ are collected at regular intervals so the sample variance $\left(s^{2}\right)$ can be computed. $s^{2}$ is also known as the plotting statistic of the chart and it is given by

$$
S^{2}=\frac{1}{n-1} \sum_{j=1}^{n}\left(X_{j}-\bar{X}\right)^{2}
$$

where $x_{j}$ is the $j$-th observation of the quality characteristic of each sample being collected at regular intervals $(j=1,2, \ldots, n) . X_{j}$ is considered normally distributed with mean $\mu_{0}$ and variance $\sigma^{2}, n$ is the size of each sample being collect at regular intervals and $\bar{X}$ is the sample mean of each sample given by

$\bar{X}=\frac{1}{n} \sum_{j=1}^{n} X_{j}$

Note that $\sigma^{2}$ is the actual variance of the process (the one which is being monitored). Here we assume that the process mean remains at the in-control value $\left(\mu_{0}\right)$ and in the exact middle point between the specification limits, consistently with the purpose of detecting relevant increases in the process variance only.

The plotting statistic $\left(S^{2}\right)$ given by Equation 1, should be compared with the Upper Control limit $\left(U C L_{S^{2}}\right)$ of the $S^{2}$ Control Chart which is given by

$U C L_{S^{2}}=\sigma_{0}^{2} \frac{\chi_{n-1,1-\alpha}^{2},}{n-1}$,

where $\sigma_{0}^{2}$ is the nominal in-control process variance, $\chi_{n-1,1-\alpha}^{2}$ is the $(1-\alpha)$-quantile of a chi-square distribution with $n-1$ degrees of freedom and $\alpha$ is the nominal false alarm rate (or in other words, the false alarm probability) chosen by the practitioner (usually, $\alpha=0.0027$ ).

A false alarm is defined as a signal (alarm) when the process is in control. The maximum false alarm rate happens when $\sigma^{2}=\sigma_{0}^{2}$. So, note that the Control Limits given by Equation 3 is 
derived in order to provide a maximum false alarm rate equal to $\alpha$, as shown in Equations 4 and 5 below.

Maximum False Alarm Rate $=1-P\left(S^{2}<U C L_{S^{2}} \mid \sigma^{2}=\sigma_{0}^{2}\right)$

$=1-P\left(S^{2}<\sigma_{0}^{2} \frac{\chi_{n-1,1-\alpha}^{2}}{n-1} \mid \sigma^{2}=\sigma_{0}^{2}\right)$

$=1-P\left(\frac{(n-1) S^{2}}{\sigma_{0}^{2}}<\sigma_{0}^{2} \frac{(n-1) \chi_{n-1,1-\alpha}^{2}}{\sigma_{0}^{2}(n-1)}\right)$,

where $\frac{(n-1) S^{2}}{\sigma_{0}^{2}}=\chi_{n-1}^{2}$ is a random variable that follows a chi-squared distribution with $n-1$ degrees of freedom, so

Maximum False Alarm Rate $=1-P\left(\chi_{n-1}^{2}<\chi_{n-1,1-\alpha}^{2}\right)=\alpha$

When the actual variance of the process $\left(\sigma^{2}\right)$ is exactly at the in-control process variance $\left(\sigma_{0}^{2}\right)$ value, the proportion of non-conforming units being produced should be small. In other words, the probability of the quality characteristic $(X)$ be smaller than the lower specification limits (LSL) or larger than the upper specification limits (USL), should be small. Figure 2 illustrates this situation. Note that these specification limits are provided by the project/manager.

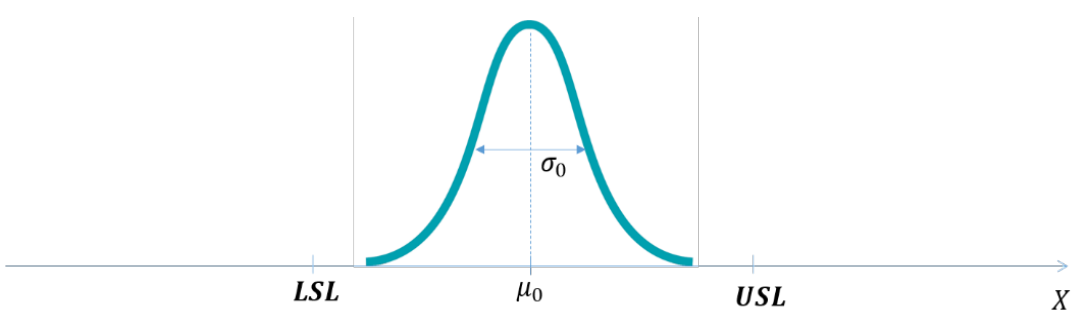

Figure 2. Process running with the nominal in-control variance $\left(\sigma^{2}=\sigma_{0}^{2}\right)$ with all the item being produced within the specification limits. Source: The authors themselves.

The $S^{2}$ Control Chart is designed to detect increases (larger than $\sigma_{0}^{2}$ ) of any magnitude in the actual process variance $\left(\sigma^{2}\right)$, even increases that does not affect the rate of nonconforming items being produced. These increases will tend to produce a signal (alarm) on the control chart. Consider the illustration provided by Figure 3 where the actual process variance is larger than $\left.\sigma_{0}^{2}\left(\sigma^{2}=\sigma_{1}^{2}>\sigma_{0}^{2}\right)\right)$, but yet the rate of non-conforming items is still small.

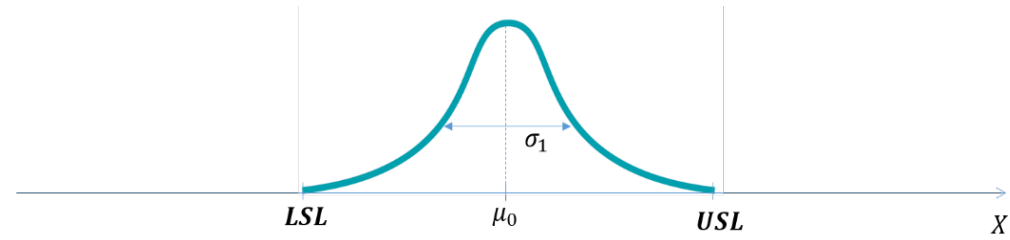

Figure 3. Process running with a variance $\left(\sigma_{1}^{2}\right)$ larger than $\sigma_{0}^{2}$, but still with all the item being produced within the specification limits. Source: The authors themselves. 
Note that since $\sigma_{1}^{2}$ is larger than $\sigma_{0}^{2}$, from the perspective of the $S^{2}$ Control Chart, the process should be declared out of control. In this case, the chart will tend to signal an alarm. However, this may be a problem because, as can be seen in Figure 3, the process is still not producing a large number of non-conforming items (almost all the items being produced are still within the specification limits even though $\sigma^{2}=\sigma_{1}^{2}>\sigma_{0}^{2}$. So, trying to fix this increase on the variance may be a waste of time and money, since in most of the cases, the process would have to be paused. So, as seen in the Introduction, it is of interest to monitor the process variance with a control chart with a broader definition of "in-control" which considers the specification limits. In the next section, we develop such kind of Control Chart for variance, we named this chart as the $S^{2}$ Modified Control Chart, in consonance with the Modified Control chart for monitoring the process mean introduced by Hill (1956).

\section{THE $S^{2}$ MODIFIED CONTROL CHART MODEL}

As discussed in the Introduction, the main idea of the chart we are proposing in this paper is that the process variance $\left(\sigma^{2}\right)$ is allowed to be larger than the in-control variance value $\left(\sigma_{0}^{2}\right)$ until a maximum value ( $\sigma_{M A X}^{2}$ ), as long as the process remains capable, in the sense that it produces a specified (tolerated) small fraction of non-conforming items $(\gamma)$. In the situation we are concerned with, instead of the in-control situation be represented by $\sigma^{2} \leq \sigma_{0}^{2}$ (where $\sigma_{0}^{2}$ represents the specified in-control target value for the process variance) as in the traditional process control setting, we allow the process to be "roughly in-control" or acceptable when $\sigma^{2} \leq \sigma_{M A X}^{2}$ (where $\sigma_{0}^{2} \leq \sigma_{M A X}^{2}$ ). If $\sigma^{2}$ assumes a value larger than $\sigma_{M A X}^{2}$, the process is deemed out-of-control (OOC). Figure 4 illustrates this situation.

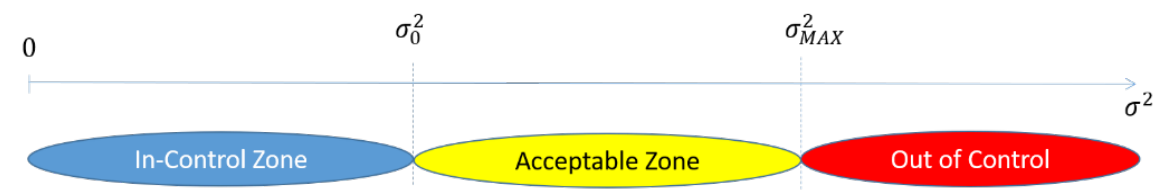

Figure 4. In-Control Zone, Acceptable Zone and Out of control Zone of the Modified Control Chart. Source: The authors themselves.

The $\sigma_{M A X}^{2}$ value must be chosen with care, depending on the lower and upper specification limits, LSL and USL, respectively, and the maximum rate (probability) of nonconforming units produced (denoted here by $\gamma$ ) that may be tolerated (or allowed). LSL, USL and $\gamma$ are specified by the management/project and have the following relationship:

$\gamma=1-P\left(L S L<X<U S L \mid \sigma^{2}=\sigma_{M A X}^{2}\right)$

where $X$ is the quality characteristic of the process and follows a normal distribution with mean $\mu_{0}$ and variance $\sigma^{2}$. As considered in the traditional $S^{2}$ Control Chart, it is assumed that the process mean remains at the in-control value $\left(\mu_{0}\right)$.

So, in other words, $\gamma$ is the maximum tolerated probability of $X$ being smaller than the LSL or greater than the USL that can be tolerated in a specific application. Figure 5 illustrates this situation where the process is running at the maximum allowed tolerated rate of non-conforming units $(\gamma)$, which happens when $\sigma^{2}=\sigma_{M A X}^{2}$. Note that if $\sigma^{2}>\sigma_{M A X}^{2}$ the rate of non-conforming units produced will be larger than the specified $\gamma$, and consequently, the process will be declared OOC. 


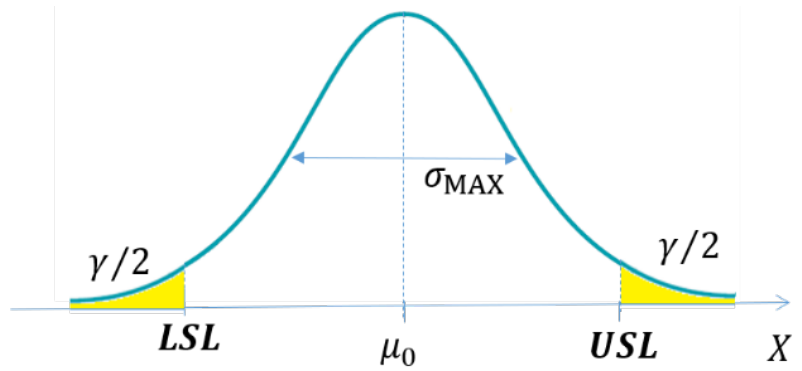

Figure 5. Process running at the maximum rate of non-conforming units being produced $\left(\sigma^{2}=\sigma_{\text {MAX }}^{2}\right)$.

Source: The authors themselves.

The maximum tolerated variance $\left(\sigma_{M A X}^{2}\right)$ can be calculated given the specification limits and the maximum tolerated rate of non-conforming units $(\gamma)$. From Figure 5, one can write

$\frac{\gamma}{2}=1-P\left(X<U S L \mid \sigma^{2}=\sigma_{M A X}^{2}\right)=1-P\left(\frac{X-\mu_{0}}{\sigma_{M A X}}<\frac{U S L-\mu_{0}}{\sigma_{M A X}}\right)$

$\frac{\gamma}{2}=1-P\left(Z<\frac{U S L-\mu_{0}}{\sigma_{M A X}}\right)=1-\Phi\left(\frac{U S L-\mu_{0}}{\sigma_{M A X}}\right)$

where $Z$ is a random variable that follows a standard normal distribution and $\Phi\left({ }^{*}\right)$ is the cumulative distribution function (c.d.f.) of a standard normal random variable. From Equation 7, one has:

$\frac{U S L-\mu_{0}}{\sigma_{M A X}}=\Phi^{-1}\left(1-\frac{\gamma}{2}\right)=z_{1-\gamma / 2}$,

where $\Phi^{-1}\left(1-\frac{\gamma}{2}\right)=z_{1-\gamma / 2}$ is the $\left(\frac{\gamma}{2}\right)$-quantile of a standard normal distribution. Since, the normal distribution is symmetric around $\mu_{0}$, one can write $U S L-\mu_{0}=\frac{U S L-L S L}{2}$, so, $\sigma_{M A X}$ can be calculated as:

$\sigma_{M A X}=\frac{U S L-L S L}{2 z_{1-\gamma / 2}}$

Equation 9 is useful because in practice what is usually defined by the project/manager is the specification limits (USL and $L S L$ ) and the maximum allowed rate of non-conforming units $(\gamma)$.

To calculate the upper control limits ( $U C L_{M o d}$ ) of the $S^{2}$ Modified Control Chart, one just need to replace $\sigma_{0}^{2}$ in the original control limit equation of the $S^{2}$ Control Chart (see Equation 3) by $\sigma_{M A X}^{2}$, as shown below:

$U C L_{M o d}=\sigma_{M A X}^{2} \frac{\chi_{n-1,1-\alpha}^{2}}{n-1}=\frac{(U S L-L S L)^{2} \chi_{n-1, I-\alpha}^{2}}{4(n-1)\left(z_{l-\gamma / 2}\right)^{2}}$. 
The plotting statistic of the $s^{2}$ Modified Control Chart is equal to the plotting statistic of the well-known $s^{2}$ Control Chart, which is given by Equation 1. Therefore, the control limit showed in Equation 10 is designed so that the maximum false alarm rate (which now happens when $\sigma^{2}=\sigma_{M A X}^{2}$ ) is actually $\alpha$. This can be shown replacing $\sigma_{0}^{2}$ by $\sigma_{M A X}^{2}$ in Equations 4 and 5 , as presented below.

Maximum False Alarm Rate $=1-P\left(S^{2}<U C L_{\text {Mod }} \mid \sigma^{2}=\sigma_{M A X}^{2}\right)$

$$
\begin{aligned}
& =1-P\left(S^{2}<\sigma_{M A X}^{2} \frac{\chi_{n-1,1-\alpha}^{2}}{n-1} \mid \sigma^{2}=\sigma_{M A X}^{2}\right) \\
& =1-P\left(\frac{(n-1) S^{2}}{\sigma_{M A X}^{2}}<\sigma_{M A X}^{2} \frac{\chi_{n-1,1-\alpha}^{2}}{(n-1)} \frac{(n-1)}{\sigma_{M A X}^{2}}\right),
\end{aligned}
$$

where $\frac{(n-I) S^{2}}{\sigma_{M A X}^{2}}=\chi_{n-1}^{2}$ is a random variable that follows a chi-squared distribution with $n-1$ degrees of freedom, so

Maximium False Alarm Rate $=1-P\left(\chi_{n-1}^{2}<\chi_{n-1,1-\alpha}^{2}\right)=\alpha$

Note that $\sigma_{I}^{2}$ illustrated in Figure 3, is exactly in the Acceptable Zone showed in Figure 4 (i.e., $\sigma_{0}^{2} \leq \sigma_{1}^{2} \leq \sigma_{M A X}^{2}$ ). So, differently from the well-known $s^{2}$ Control Chart, the $s^{2}$ Modified Control Chart will not tend to signal an alarm when $\sigma^{2}=\sigma_{l}^{2}$. This is desirable, since when $\sigma^{2}=\sigma_{l}^{2}$ the process is still not producing an unacceptable rate of non-confirming units. In the next section, we provide an illustrative example showing the advantages of the proposed $s^{2}$ Control Chart in the case illustrated in Figure 3 (i.e., in the case of $\sigma^{2}=\sigma_{l}^{2}$ ).

\section{AN ILLUSTRATIVE EXAMPLE}

We illustrate the ideas of the $s^{2}$ Modified Control Chart proposed in this paper in an automobile engine manufacturing process that uses a forging process to make piston rings. A more detailed description of this example is given in Montgomery (2009, p. 251). The quality characteristic variable $(X)$ is the internal diameter of the piston rings, which has a two-sided specification limits of $74.000 \pm 0.050 \mathrm{~mm}$. It is assumed that the piston rings diameter $(X)$ follows a normal distribution. Different from the book, here we assume that the in-control mean $\left(\mu_{0}\right)$ and the in-control standard deviation $\left(\sigma_{0}\right)$ of the piston rings diameter are known, being respectively $74.000 \mathrm{~mm}$ and $0.0100 \mathrm{~mm}$.

The process leadership defined as acceptable up to 96 non-conforming parts per million (ppm) of units produced. In other words, the maximum allowed rate of non-conforming items $(\gamma)$ is $96 / 1000000=0.000096$, which provides $z_{l-\gamma / 2}=3.9$. So, the maximum standard-deviation allowed $\left(\sigma_{M A X}\right)$ for the piston ring diameter can be calculated using Equation 9, as shown below.

$\sigma_{M A X}=\frac{U S L-L S L}{2 z_{1-\gamma / 2}}=\frac{74.05-73.95}{2 \times 3.9}=\frac{0.1}{7.8}=0.0128$

All parameters given by this example are summarized in Table 1. 
Table 1. Parameters provided by the Example

\begin{tabular}{cccccc}
\hline$\mu_{0}$ & $\sigma_{0}$ & $\sigma_{\text {MAX }}$ & USL & LSL & $\gamma$ \\
\hline 74.000 & 0.0100 & 0.0128 & 74.050 & 73.950 & 0.000096 \\
\hline
\end{tabular}

Source: The authors themselves.

Suppose that the practitioner decides to monitor the process variance $\left(\sigma^{2}\right)$ with samples of size $n=5$ of the piston's rings diameter collected at regular intervals. To this end, the practitioner can use the well-known $s^{2}$ Control Chart or the $s^{2}$ Modified Control Chart proposed here. Considering a maximum false alarm rate $(\alpha)$ of 0.0027 for each chart, the control limits of both charts ( $U C L_{S^{2}}$ and $U C L_{\text {Mod }}$ ) can be calculated as shown below.

$U C L_{S^{2}}=\sigma_{0}^{2} \frac{\chi_{n-1,1-\alpha}^{2}}{n-1}=0.0100^{2} \frac{16.25}{5-1}=0.000406$

$U C L_{M o d}=\sigma_{M A X}^{2} \frac{\chi_{n-1,1-\alpha}^{2}}{n-1}=0.0128^{2} \frac{16.25}{5-1}=0.00067$

Now, let's suppose that the process standard deviation $(\sigma)$ moved from the in-control value $\sigma=\sigma_{0}=0.0100$ to $\sigma=\sigma_{1}=0.0114$. Note that, since $\sigma_{0}<\sigma_{1}<\sigma_{M A X}$, even though the process standarddeviation increased, it is still in the Acceptable Zone (see Figures 3, 4 and 6), so the process is still producing an acceptable rate of non-conforming items (i.e., a rate smaller than $\gamma=0.000096$ ).

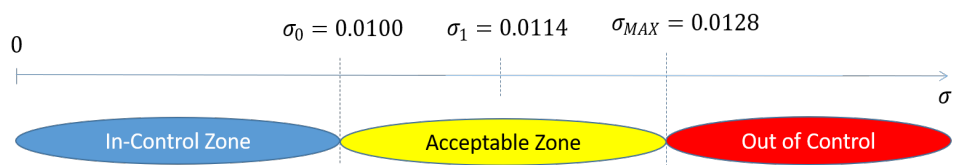

Figure 6. Illustration showing the actual process standard deviation ( $\sigma$ ) in the Acceptable Zone ( $\sigma=\sigma_{l}=0.0114$ ). Source: The authors themselves.

To further examine the behavior of both charts (the well-known $s^{2}$ Control Chart and the $S^{2}$ Modified Control Chart proposed here), we simulate one thousand independent samples of the piston ring diameter (each sample with size 5), from a normal distribution with mean $\mu_{0}=74.000$ and standard deviation $\sigma=\sigma_{l}=0.0114$. With the simulated data, we calculated the sample variances $\left(s^{2}\right)$ using Equation 1, which is the plotting statistic for both charts, and plotted these against the control limits values shown in Equations 14 and 15. The simulated sample variances $\left(S^{2}\right)$ and the control limits $\left(U C L_{S^{2}}\right.$ and $U C L_{M o d}$ ) are shown in Figure 7. $U C L_{S^{2}}$ is shown in a dashed grey line and $U C L_{M o d}$ in a solid black line.

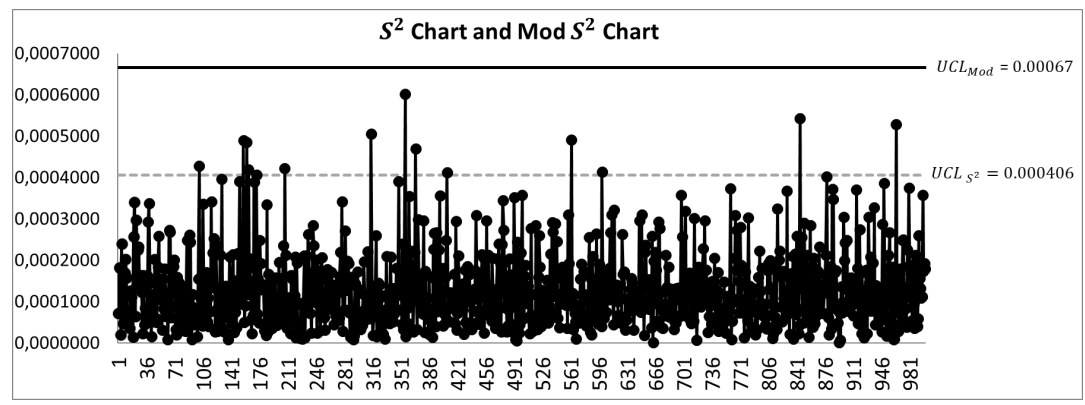

Figure 7. The $S^{2}$ Control Chart and the $S^{2}$ Modified Control Chart for monitoring the variance of a process $X \sim N\left(\mu_{0}=74, \sigma=\sigma_{l}=0.0114\right)$ given the in-control parameters in Table 1. Source: The authors themselves. 
As it can be seen in Figure 7, signals above the $U C L_{S^{2}}$ dashed line are frequent. If the user were using just the well-known $S^{2}$ Control Chart, he would typically suspect that an assignable cause has occurred and that the process variance $\left(\sigma^{2}\right)$ is larger than the incontrol target $\left(\sigma_{0}^{2}=0.01^{2}\right)$ increasing the production of non-conforming items. In this case, the user would stop the process and start looking for assignable causes, wasting time and decreasing production (what is also a waste of money). However, even though the process variance has indeed increased to $\sigma^{2}=\sigma_{1}^{2}=0.0114^{2}$, it is still smaller than the maximum variance allowed $\left(\sigma_{M A X}^{2}=0.0128^{2}\right)$, this means that the proportion of non-conforming items being produced is still acceptable according to the specification of the project. Therefore, the production does not really need to be stopped to search for assignable causes. So, it is clear that the use of the well-known $S^{2}$ Control Chart alone can mislead the user.

Now, consider the $S^{2}$ Modified Control Chart proposed in present paper, since the process is still capable in the sense it is still producing an acceptable rate of non-conforming units, there is no signal above the $U C L_{M o d}$ black solid line, what is indeed expected since the probability of a false alarm when $\sigma^{2}=\sigma_{1}^{2}=0.0114^{2}$ is smaller than $\alpha=0.0027$, as shown below.

False Alarm Rate $=1-P\left(S^{2}<U C L_{\text {Mod }} \mid \sigma^{2}=\sigma_{1}^{2}=0.0114^{2}\right)$

$$
\begin{aligned}
& =1-P\left(S^{2}<\sigma_{M A X}^{2} \frac{\chi_{n-1,1-\alpha}^{2}}{n-1} \mid \sigma^{2}=\sigma_{1}^{2}=0.0114^{2}\right) \\
& =1-P\left(\frac{(n-1) S^{2}}{\sigma_{I}^{2}}<\sigma_{M A X}^{2} \frac{\chi_{n-1,1-\alpha}^{2}}{(n-1)} \frac{(n-1)}{\sigma_{I}^{2}}\right)
\end{aligned}
$$

where $\frac{(n-1) S^{2}}{\sigma_{1}^{2}}=\chi_{n-1}^{2}$ is a random variable that follows a chi-squared distribution with $n-1$ degrees of freedom, so

False Alarm Rate $=1-P\left(\chi_{n-1}^{2}<\frac{\sigma_{M A X}^{2}}{\sigma_{l}^{2}} \chi_{n-1,1-\alpha}^{2}\right)$

$=1-P\left(\chi_{n-1}^{2}<\frac{0.0128^{2}}{0.0114^{2}} 16.25\right)$

$=1-P\left(\chi_{n-1}^{2}<20.486\right)=0.0004<\alpha=0.0027$.

The large number of signals between $U C L_{S^{2}}$ and $U C L_{M o d}$ control limits when the actual process variance $\left(\sigma^{2}\right)$ is in between $\sigma_{0}^{2}$ and $\sigma_{M A X}^{2}$ (as shown here in the case of $\sigma^{2}=\sigma_{l}^{2}$ ) and the smaller frequency of signal above $U C L_{\text {Mod }}$ given the small value of the false alarm rate in this case (see Equation 17), motivates the use of the $s^{2}$ Modified Control Chart proposed in the present paper.

In summary, as $\sigma^{2}$ moves from $\sigma_{0}$ towards $\sigma_{M A X}$, the chart tends to more quickly signal points higher than $U C L_{S^{2}}$ and not higher than $U C L_{\text {Mod }}$, since the process is still capable. If the process had been monitored only by using the $S^{2}$ control chart (i.e., only by using the $U C L_{S^{2}}$ control limit), it would have signaled several alarms, however, that do not compromise the 
process in meet its quality requirements, which could keep running in order to fulfill the process expectancies in terms of efficiency and quality.

\section{CONCLUSION}

In this paper, we introduced a new control chart to monitor the variance of quality characteristics being produced by capable processes. Differently from the well-known $s^{2}$ Control Chart, this new tool considers, in its formulation, the process specifications limits provided by the project/manager and not only the nominal in-control process variance. We named this new chart as the $s^{2}$ Modified Control Chart, since it is a natural extension of the Modified Control Chart for monitoring the process mean presented in Montgomery (2009) and introduced by Hill (1956).

Differently from the $s^{2}$ Control Chart, the $s^{2}$ Modified Control Chart detects only genuinely increases in the process variance, which significantly increase the rate of nonconforming items being produced. This prevents the practitioner to stop the process and look for assignable causes if only a small increase in the process variance occurs. This is desirable in the sense that small increases in the variance may not affect much the rate of notconforming items being produced and pausing the process generate extra costs. We illustrated the ideas of $s^{2}$ Modified Control Chart in an example with simulated data.

Finally, we emphasize that the present work is part of a working in progress in which we are studying the power of the proposed $s^{2}$ Modified Control Chart and its performance when used jointly with the Modified Control Chart for monitoring the process mean and when it is designed with estimated parameters.

\section{REFERENCES}

Freund, R.A. (1957), "Acceptance control charts", Industrial Quality Control, Vol. 14, No. 4, pp. 13-23.

Hill, D. (1956), "Modified control limits", Applied Statistics, Vol. 5, No. 1, pp. 12-9.

Mohammadian, F. and Amiri, A. (2012), "Economic-statistical design of acceptance control chart", Quality and Reliability Engineering International, Vol. 29, No. 1, pp. 53-61.

Montgomery, D.C. (2009), Introduction to Statistical Quality Control, John Willey \& Sons, New York.

Oliveira, B.K.S., Jardim, F.S., Chakraborti, S. et al. (2018), “Effects of Parameter Estimation on the Modified and Acceptance Control Charts", in Joint Statistical Meeting, Proceedings Paper. Denver, USA. American Statistical Association.

Woodall, W.H. and Faltin, F.W. (2019), "Rethinking control chart design and evaluation", Quality Engineering, Vol. 31, No. 4, pp. 596-605.

Wu, Z. (1998), "An adaptive acceptance control chart for tool wear", International Journal of Production Research, Vol. 36, No. 6, pp. 1571-86.

Author contributions: All authors contributed equally to this paper. 